\title{
DEFINING DENSITIES FOR URBAN RESIDENTIAL TEXTURE, THROUGH LAND USE CLASSIFICATION, FROM LANDSAT TM IMAGERY: CASE STUDY OF SPANISH MEDITERRANEAN COAST
}

\author{
N. Colaninno ${ }^{\mathrm{a}, *}$, J. Roca $^{\mathrm{a}}$, M. Burns ${ }^{\mathrm{a}}$, B. Alhaddad $^{\mathrm{a}}$ \\ ${ }^{a}$ CPSV, Centre of Land Policy and Valuations of the Department of Architectural Technology I at Universitat \\ Politècnica de Catalunya, Barcelona Tech (UPC), Av. Diagonal 649, 08028 Barcelona, Spain. \\ cpsv.info@upc.edu
}

Commission VII, WG VII/4

KEY WORDS: Land Use, Urban Patterns, Classification, Morphology, Planning, GIS, Landsat

\begin{abstract}
:
In the recent epoch, there has been considerable debate about the urban development along the European Mediterranean area, also undertaken by the European Authorities, and in particular regarding the role of spatial planning in order to improve sustainable trends of land use. Great transformations along the Spanish Mediterranean coast have generated considerable changes in the traditional structure of the landscape, far from the typical model of Mediterranean cities, and the rapidity of these modern dynamics has been a significant impact on the spatial patterns, also associated with the expansion of urban connections through the whole territory. The increase of large peri-urban areas, sprawled on the territory, and caused by uncontrolled, uncoordinated and unplanned growth, inevitably has brought the cancellation of clearly identifiable boundaries between the city and the rural areas. Spatial analysis, within quantitative geography and linked to the emerging field of regional science, represents a synthesis of urban and regional economics that is consistent with the complex sciences which dominate the simulation of urban form and functions. Most urban models deal with the city in terms of the location of its economic and demographic activities, but there is also a move to link such models to urban morphologies (Batty 2008). According with these concepts, the investigation, also supported by the use of technologies such as remote sensing and GIS, aims to complement the spatial analysis of regional development dynamics by classifying urban structures and quantifying some of main characteristics based on morphological features.
\end{abstract}

\section{INTRODUCTION}

\subsection{Overview and motivations}

During the last decades, Spain has been undergoing important processes of urban growth, which has implied the consumption of large amounts of land, although the total population has hardly increased. This consequence has been very important along the coastal territory of Spanish Mediterranean side, where modern and actual dynamics of development are requiring new ways to analyze and quantify urban growth phenomena. Urban settlements, and their own formal characteristics, have to be wholly defined, being this basic information essential in spatial planning, in order to prepare the best practices apt to "respond" to the current challenges of territorial changes. How to extract and analyze information about residential settlements quickly and objectively is the foundation of the studies about economic and social development. The improvement of remote sensing technology provides a rapid tool for acquiring such information quickly (Chen et al. 2010). Quite a lot of useful techniques based on remote sensing technologies and methodologies for automatic classification of urban landscape have been developed, but there is still a challenge to find a generalized and objective methodology to be applied in different situations and geographical contexts. The use of automatic processes requires the reduction, as much as possible, of subjective interpretations and arbitrary interventions of analysts.

\subsection{General objectives}

The investigation aims to find a relative fast and objective methodology to extract impervious areas on large landscapes and define different types of urban models, depending on morphological characteristics of land occupation. The work pretend to strengthen the idea that new technologies can really support all the process of planning both in the detection phase than in the analysis, and in order to support all the decisions which bring the final drafting of spatial plans.

\subsection{Specific objectives}

First pursued result is the classification of several land cover categories along the Mediterranean side of Spain, through the use of satellite imagery and remote sensing techniques. It will be place emphasis on the impervious areas. After that we pretend to improve a methodology for automatic categorization of different typologies of urban texture, depending on their physical characteristics and based on a set of indicators such as size, shape, density and fragmentation of the urban settlements. Final result will be the automatic classification of three main morphological models of urban fabric: continuous, discontinuous, and scattered. It will be achieved by using statistical techniques such as factorial and cluster analysis.

\footnotetext{
* Architect and PhD candidate in Urban and Architectural Management and Valuations at UPC, Universitat Politècnica de Catalunya, Barcelona Tech. Researcher at the LMVC, Virtual City Modelling Lab of the Universitat Politècnica de Catalunya, Av. Diagonal 649, 4 ${ }^{\text {a }}$ pl. 08028 Barcelona, Spain. Tel: 0034 934011933. E-mail: nicola.colaninno@upc.edu nicola.colaninno@gmail.com
} 


\subsection{Effectiveness of the work}

The urban environment is a very complex and dynamic context, since it involves a large number of factors which evolve continuously. On the other hand, most of the processes which develop in the framework of urban areas are connected with the physical space. Therefore, measure, analyze and understand the dynamic processes and their changes along the time, it is critical to generate accurate spatial and temporal information. It is necessary that the spatial information could allow the more precise estimation of the developing phenomena of the urban areas over a territory. It is important the estimation the rate of growth in terms of consumption of natural resources, but it is also required more detailed information on the morphological characteristics of the urban fabric in order to outline different patterns of spatial growth and to make it possible to estimate which kind of urban settlement is moving towards a sustainable model of development. Even more important is to use automated tools that allow rapid and detailed analysis over huge areas and in different geographical contexts. In this framework the investigation aims to suggest possibilities to improve analytical tools for analysis and management of the urban and natural landscape, also supporting the processes of planning with data continuously updating.

\section{LAND COVER CLASSIFICATION THROUGH REMOTE SENSING TECHNIQUES APPLIED ON LANDSAT 4-5 TM IMAGERY}

\subsection{Analyzed data}

Data source is provided through the use of USGS Glovis webpage, and based on Landsat 4-5 TM collection for the year $2011^{*}$. In general, Landsat satellite provides multi-spectral images, at $30 \mathrm{~m}$ of resolution and at different wavelengths, thermal images at $60 \mathrm{~m}$ of resolution and panchromatic images at $15 \mathrm{~m}$ of resolution. The satellite uses three primary sensors that have evolved over more than thirty years: MSS (Multispectral Scanner), TM (Thematic Mapper) and ETM+ (Enhanced Thematic Mapper Plus). Table 1 shows with more details the main characteristics for all the sensors of Landsat.

\begin{tabular}{|c|c|c|c|c|c|}
\hline $\begin{array}{l}\text { Landsat } \\
\text { Satellite }\end{array}$ & Sensor & Band & $\begin{array}{c}\text { Spectral } \\
\text { Range }\end{array}$ & Scene Size & $\begin{array}{c}\text { Pixel } \\
\text { Res }\end{array}$ \\
\hline L 1-4 & $\begin{array}{l}\text { MSS multi- } \\
\text { spectral }\end{array}$ & $1,2,3,4$ & $0.5-1.1 \mu \mathrm{m}$ & \multirow{6}{*}{$\begin{array}{c}185 \mathrm{X} 185 \\
\mathrm{~km}\end{array}$} & $\begin{array}{c}60 \\
\text { meter }\end{array}$ \\
\hline L 4-5 & $\begin{array}{c}\text { TM multi- } \\
\text { spectral }\end{array}$ & $1,2,3,4,5,7$ & $0.45-2.35 \mu \mathrm{m}$ & & $\begin{array}{c}30 \\
\text { meter }\end{array}$ \\
\hline L 4-5 & TM thermal & 6 & $\begin{array}{c}10.40-12.50 \\
\mu \mathrm{m}\end{array}$ & & $\begin{array}{c}120 \\
\text { meter }\end{array}$ \\
\hline L 7 & $\begin{array}{l}\text { ETM+ multi- } \\
\text { spectral }\end{array}$ & $1,2,3,4,5,7$ & \begin{tabular}{|c}
$0.450-2.35$ \\
$\mu \mathrm{m}$
\end{tabular} & & $\begin{array}{c}30 \\
\text { meter }\end{array}$ \\
\hline L 7 & ETM+ thermal & $6.1,6.2$ & \begin{tabular}{|c|}
$10.40-12.50$ \\
$\mu \mathrm{m}$
\end{tabular} & & $\begin{array}{c}60 \\
\text { meter }\end{array}$ \\
\hline anchromatic & ETM+ thermal & 8 & $0.52-0.90 \mu \mathrm{m}$ & & $\begin{array}{c}15 \\
\text { meter }\end{array}$ \\
\hline
\end{tabular}

Table 1. Main characteristics for Landsat satellite imagery. GLCF Global Land Cover Facility

The images are downloaded in GeoTIF format and the pixel is identified with a Digital Number (DN) on a scale of 0 to 255 . We have calibrated the images in order to convert the DN in a value of reflectance which provides values on a scale of 0 to 1 . After calibration process, it was proceed to mosaic all the necessary imagery apt to cover each of the analyzed areas.

\footnotetext{
* C LANDSAT Image Copyright 2011, USGS
}

Together with the multispectral images based on bands 1, 2, 3, 4,5 , and 7 of Landsat TM 4-5, it was used for this study the Digital Elevation Models (DEM) with a resolution of $30 \mathrm{~m}$, to provide the physical characteristics of the territory.

\subsection{Premise}

Spatial resolution, spectral information and advanced processing techniques are important in order to get the best results from satellite imagery analysis. One of the main parts of this investigation is focused on enhance the spectral information through the generation of additional layers (in addition to the original information provided by Landsat sensors) in order to minimize the mistakes of classification processes. Actually if we work with the six bands of Landsat, we will get lot of problems in the results of classification, mostly due to mixing characteristics of land cover classes, and in particular between soils, and impervious classes. It is because the spectral characteristics of these classes seem to be quite similar in certain wavelengths. While vegetation results the most obvious information in the remote imagery. The leaf of plant exhibits a strong absorption property in the red band and a strong reflectance in the NIR. The reflection reduces slightly from green band to red band and then a reflection valley is generated. The reflection rose sharply in the NIR and a reflection peak is formed; a valley again in the SWIR for the reflection weakens rapidly ( $\operatorname{Lin}$ et al. 2010). Water shows the highest reflectance values at the band 1, i.e. the blue band, whereas gradually decreasing in successive bands to reach the lowest values at the SWIR bands (Figure 1).

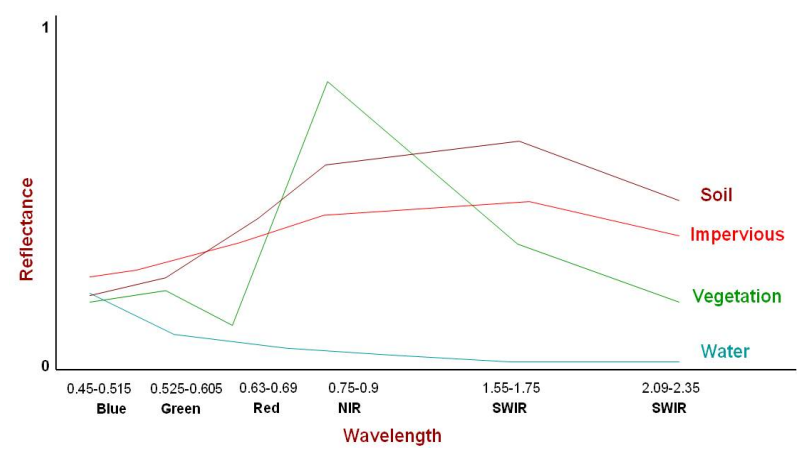

Figure 1. Trend of Spectral characteristics for four land cover classes in the case of Landsat 4-5 sensor

Based on the study of the spectral characteristics of the material, a lot of techniques for specific material abundance detection and indices of defined characters have been developed until now. We have taken advantage of these instruments to generate a multi-indices image, based on 28 indices to reduce the mistakes due to the most common classification techniques.

\subsection{Methodology}

2.3.1 Building a Multi-index image: Previous treatments have been applied such as calibration, to get reflectance values from the digital numbers (DN) of the GeoTIF images, and atmospheric correction by using Quick Atmospheric method. Several images were "mosaicked" together, in order to cover the areas under investigation which, in our case, refer to the administrative boundaries of the Autonomous Communities along the Spanish Mediterranean coast. After that it has been used several band transformation procedures to extract single 
image bands which make in evidence the abundance of specific categories of land cover types such as vegetation, built-up area, wet lands, etc., in order to reduce the effects of environment and then the "noise" due to the mixture between different classes. These procedures also provide subtle spectralreflectance or colour differences between surface materials that are often difficult to detect in a standard image. Linear Spectral Unmixing (LSU) technique has been used to determine the relative abundance of specific categories of coverage of the soil, according to the spectral characteristics of the materials, through the use of the original multispectral images composed of six bands (blue, green, red, near infrared and two middle infrared bands). The reflectance of a pixel in the image is considered as a linear combination of the reflectance of each land cover class present within the pixel itself. The larger the pixel, the more the mixture of materials occurs. Since the spectrum of a pixel a weighted average of the quantity of a material for the spectrum of the material, the use of this technique allows to generate new images in which a specific category of use of soil is discriminated and highlighted with high brightness. For this investigation, LSU it has been applied twice, based on two different spectral libraries each one based on three categories: urban, industrial, and soil (Figure 2). The LSU process provides a total of six layers.

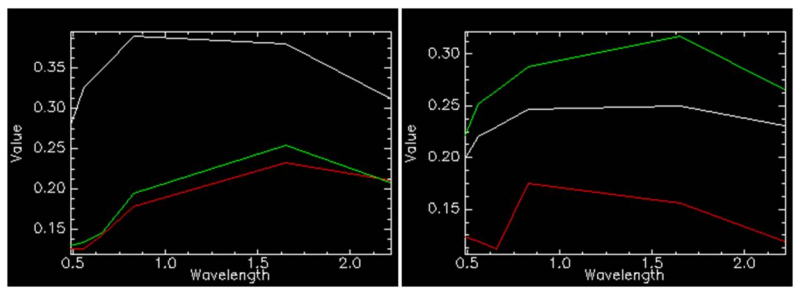

Figure 2. Spectral libraries used to make Linear Spectral Unmixing

Tasseled cap vegetation index, for Landsat TM 5 data, was employed to perform an orthogonal transformation of the original data into three factors: Brightness, Greenness, and Third. Brightness consists of the soil brightness index (SBI), Greenness is the green vegetation index (GVI), while the third component is related to soil features, including moisture status. The concept of tasseled cap transformation is a useful tool for compressing spectral data into a few bands associated with physical scene characteristics (Crist and Cicone 1984). In order to transform multispectral data into a single image band which enhances the vegetation distribution, the NDVI (Normalized Difference Vegetation Index), the Atmospherically Resistant Vegetation Index (ARVI), and the Enhanced Vegetation Index (EVI) were used. The NDVI transformation, obtained as Band4-Band3/Band4+Band3, indicates the amount of green vegetation present in a pixel. Higher NDVI values indicate more green vegetation. ARVI is an enhancement to the NDVI that is relatively resistant to atmospheric factors (for example, aerosol). It uses the reflectance in blue to correct the red reflectance for atmospheric scattering. The value of this index ranges from -1 to 1 . The common range for green vegetation is 0.2 to 0.8 (Kaufman and Tanre, 1996). EVI was developed to improve the NDVI by optimizing the vegetation signal in Leaf Area Index (LAI) regions by using the blue reflectance to correct for soil background signals and reduce atmospheric influences, including aerosol scattering. This VI is therefore most useful in LAI regions, where the NDVI may saturate. The value of this index ranges from -1 to 1 . The common range for green vegetation is 0.2 to 0.8 (Huete et al. 1997). The
Normalized Difference Soil Index (NDSI) was used because gives a more reliable estimation in a case of exposed soil conditions. This index is formulated to portray the characteristic of responses from soil other than vegetation or water (Kasimu and Tateishi 2010) and can be helpful to discriminate deciduous broad-leaved forest and dry land with sparse crop. A simple logic expression by combining 4-3 was used to enhance residential areas information, since this composite index allows an ideal effect to extract urban built-up land (XU H. Q. 2005). We found out useful another simple expression such as TM4TM3 in order to discriminate between Built-up area, soils and vegetation. The Soil Adjusted Vegetation Index (SAVI) is generally used to minimize the effects of soil background. Similarly to the Normalized Difference Vegetation Index, the near infrared and red bands are used in the calculation, but with the addition of an adjustment factor (L), which varies between zero and one (Crocetto and Tarantino 2009). According to Qi et al. (1994) for this work we have used the Modified SAVI (MSAVI) which appears to be a more sensitive indicator of vegetation amount by raising the vegetation signal and simultaneously lowering soil-induced variations. It also were used two normalized water indices: the Normalized Difference Water Index (NDWI) and the Modified NDWI. The NDWI (McFeeters 1996) works in the same manner as the NDVI transformation used to map vegetation. This index produces a single gray-scale image where water is brighter. While the NDWI works with bands 2 and 4, the modified NDWI (MNDWI) takes into account band 2 and band 5 (Xu 2006). The computation of the MNDWI will produce three results: (1) water will have greater positive values than in the NDWI as it absorbs more MIR light than NIR light; (2) built-up land will have negative values as mentioned above; and (3) soil and vegetation will still have negative values as soil reflects MIR light more than NIR light (Jensen 2004). In order to improve the discrimination between impervious land cover categories, and bare soils, it appears to be really useful the use of the texture analysis. Taking into account that, within an image are present different regions characterized by a variation of brightness, the texture analysis refers to the spatial variation of the brightness and as a function of the scale. In order that a given area can be discriminated for different characteristics of texture, the gray levels within the area must have a high level of homogeneity between them. Co-occurrence-based texture filter was used in this study, which provides mean, variance, homogeneity, contrast, dissimilarity, entropy, second moment, and correlation. Co-occurrence measures use a gray-tone spatial dependence matrix to calculate texture values. This is a matrix of relative frequencies with which pixel values occur in two neighbouring processing windows separated by a specified distance and direction. It shows the number of occurrences of the relationship between a pixel and its specified neighbour ${ }^{*}$. It is also taken into account the orographic component of the territory through the use of the Digital Elevation Model (DEM) at $30 \mathrm{~m}$ resolution, and the slope measured in degrees and calculated on the DEM. It was so obtained a final set of 28 layers also by using the three infra red bands of Landsat: Band 4, band 5, band 7, LSU 1 and LSU 2, Tasselled Cap, NDVI, ARVI, EVI, NDSI, 4-3, MSAVI, NDWI, MNDWI, Texture LSU 2, Slope, DEM. To increase the divisibility between different group objects, all the indices were stretched to the range from 0 to 255 (Lin et al. 2010), which appears to be also advantageous to visualize the different behaviours of the categories for different indices.

\footnotetext{
*ENVI help guidelines
} 
2.3.2 Spectral libraries and classification: Once we get the multi-index image, and through a process of image interpretation, we selected the categories of land cover, also taking advantage by the use of Google Hearth platform, which provides higher resolution images, in order to corroborate the categories chosen. For defining land cover classes we proceeded as follow: first of all, three possible types of urban areas were selected; afterwards the potential industrial categories, and then plenty of possible kinds of terrain. After that, it has been selected the green surfaces, and the waters depending on the degree of depth. Tools of automatic vegetation delineation and relative water depth detection were used to better identify the above categories. The surfaces were selected through the instrument of ROI, and for each ROI selected it was calculated class statistics. The mean value, for each class and at each band (previously normalized in the 0-255 scale, as mentioned before), has been used to generate a spectral library with a final set of 48 land cover classes (Figure $3-4)$. This procedure is also important to analyze the specific characteristics of the materials, depending on the indices, which is useful to make corrections at the curves to improve the following classification based on the spectral library.

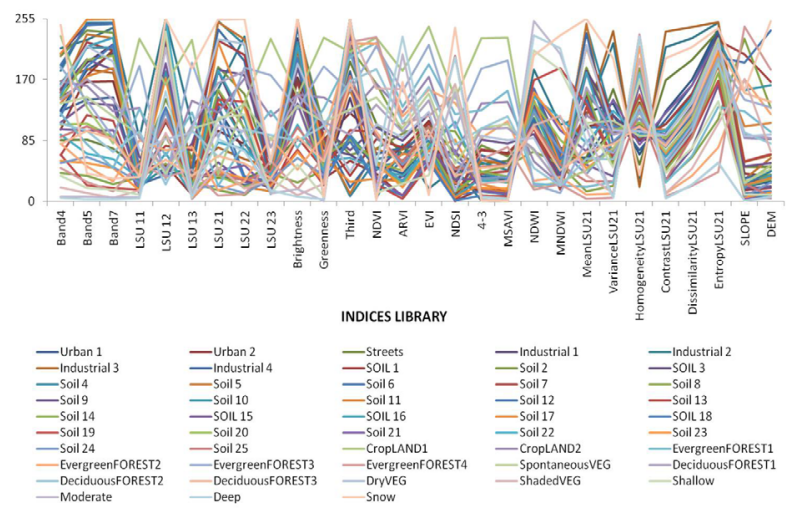

Figure 3. The spectral library, based on mean values, used to classify

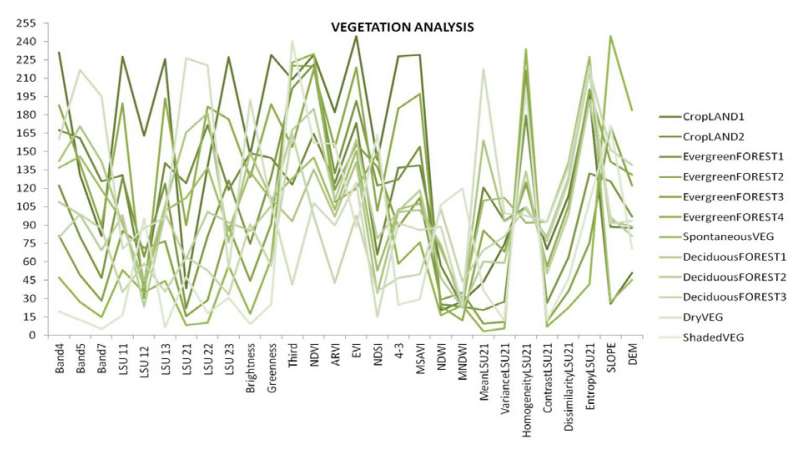

Figure 4. Example of the spectral library for vegetation classes

Automated pixel-based multispectral classification of our multiindices imagery is applied by using Minimum Distance algorithm and the spectral library above mentioned. The Minimum Distance technique calculates the Euclidean distance between each pixel within the image and the average value, for the specific class of land cover, represented in the spectral library. The classification has been repeated for all Autonomous Communities of Spanish Mediterranean coast always using the same spectral library of figure 3 , and we found out a really good homogeneity of results in the comparison between the different classifications of land cover for the Autonomous Communities. Figure 5 shows an example of the results of Minimum Distance classification and for two Autonomous Communities: Cataluña and Valencia.
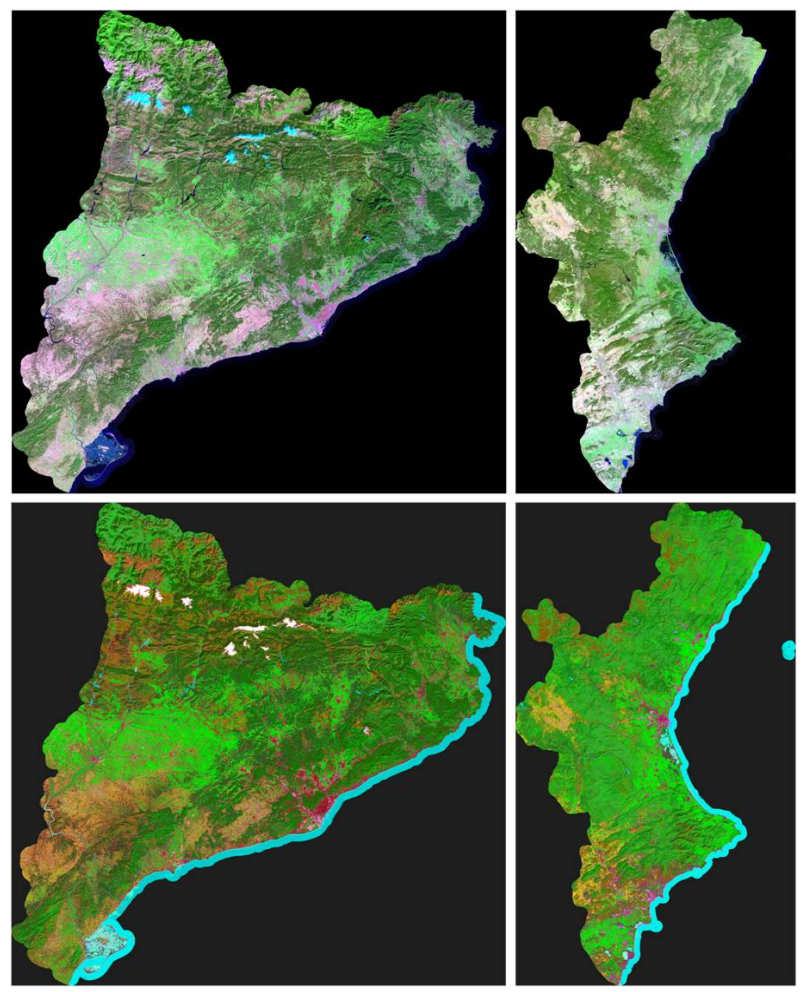

Figure 5. Land covers classification result by applying Minimum Distance technique.

\subsection{Interpretation of primary results, correction, and accuracy analysis}

Although the result of the classification provides a broad overview about the class composition into the landscape under consideration, we focused the analysis on the impervious land cover classes. Actually a review of primary results, evidences interesting outcomes in discriminating artificial surfaces and natural land cover classes such as vegetation, water, cropland or bare lands. The process also allows certain goodness in identifying different impervious typologies, such as residential and industrial settlements, even it is mixing with some categories of terrains. A second step of classification was applied in order to clean the mistakes due to upon mentioned mixture. This classification is based on parallelepiped algorithm and just aims to reclassify those terrains which mixed up with urban functions. Parallelepiped classification uses a simple decision rule to classify multispectral data. The decision boundaries form an $n$-dimensional parallelepiped classification in the image data space. The dimensions of the parallelepiped classification are defined based upon a standard deviation threshold from the mean of each selected class*. It was reclassified four types of terrains, selected by using ROI tool, and the result was overlapped on the primary classification to improve the result (Figure 6). Red tone colours indicate residential areas, while yellow and cyan show industrial estates.

\footnotetext{
${ }^{*}$ ENVI help guidelines
} 

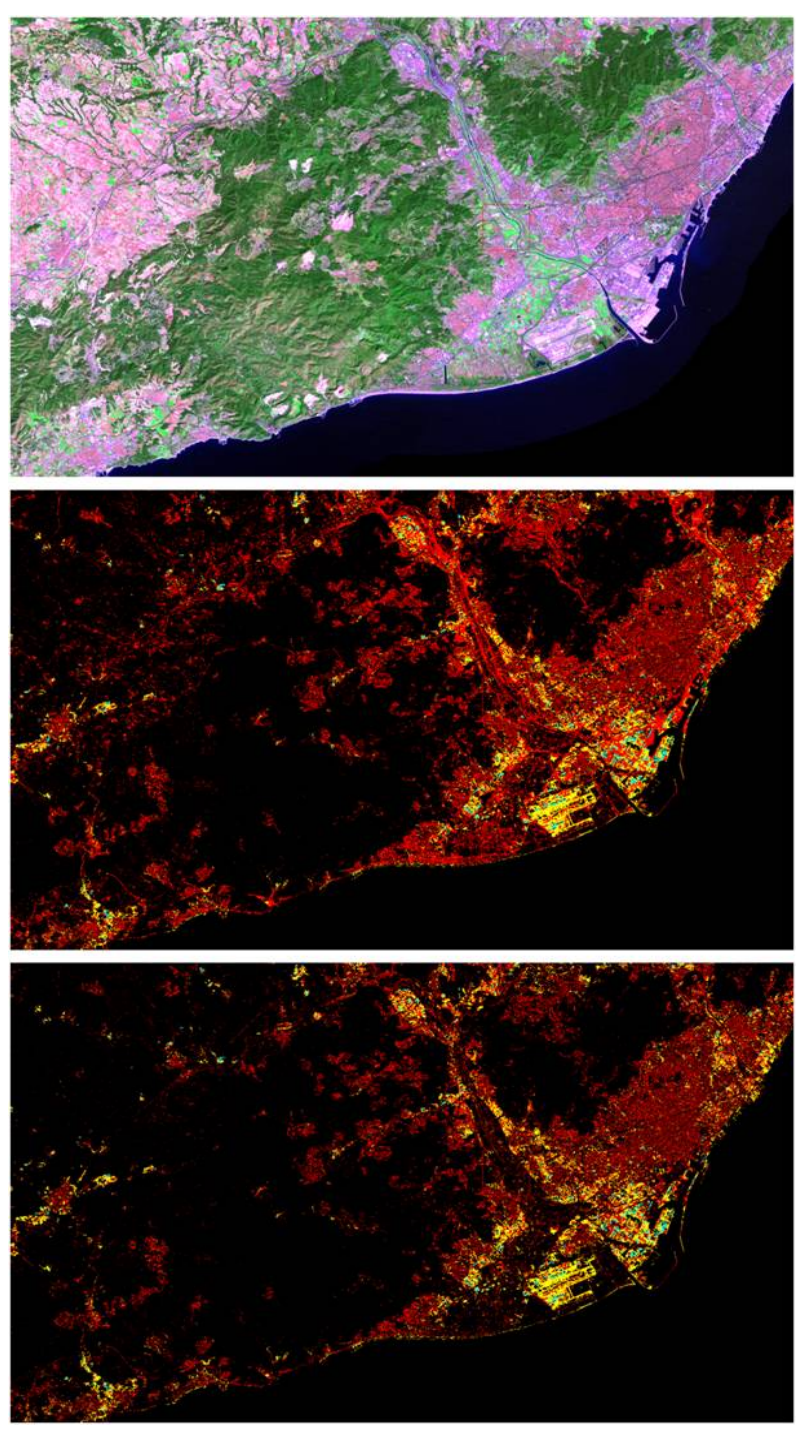

Figure 6. Landsat image; an example of result of Minimum

Distance classification, and final result after correcting

A quick analysis of validation, focused on impervious areas detected, has been realized for evaluating the goodness of the applied processes, by using a confusion matrix based on ground truth region of interest. A plenty of ROIs were selected all over the landscape under investigation, which was enhanced by using a sharpen filter to get a better visualization. Considering a single class which merges together the impervious categories found, it was reached an overall accuracy of around 63\%. This value is due to an important amount of terrains which are still mixing with urban areas, but further investigations will be focused on differentiate those kind of soils.

\subsection{First remarks}

Actually it is really difficult, at this spatial resolution, to discriminate different typologies of urban settlements and certain bare soils. That is why we look for additional layers and steps, in order to express the most effective land cover composition of urban landscape, consistent with the scale of analysis. The results of the work will be an important step, but a new starting point, to redefine a more precise spectral library and a possible different composition of the layers into the images in order to achieve the better and faster outcomes.

\section{DEFINING URBAN CLASS CATEGORIES, BASED ON MORPHOLOGICAL FEATURES}

\subsection{Overview}

The development of GIS technologies has provided a variety of analytical tools for analysis and management of landscape, urban or natural. The ability to quantify the structure of a territorial system is a basic requirement for the study of environment and its changes over time. The quantitative metric, based on descriptive indicators, provides a representative database which allows analyzing the landscape, but the interpretation of the indicators requires an adequate knowledge of the geographical context but, most of all, of the phenomenon under investigation. In this work, under the hypothesis that urban settlements are the effect of a sum of different typologies of morphological structure, we intend to automatically discriminate three different types of texture: continuous, discontinuous, and scattered.

\subsection{Methodology}

3.2.1 Post-processing the remote sensing result: Once obtained the final dataset about impervious areas, we aim to measure the degree of physical continuity of urban settlements through the use of morphological features such as shape, fragmentation, and density, in order to define strong and weak relations between the composing parts of the urban texture. After applying filters of clump and median it has been converted, the result of remote sensing classification, in a shapefile and exported to a GIS platform. Morphological features for all the patches, which compose the landscape, will be now synthesized through the use of synthetic indicators.

3.2.2 Morphological indices: Three synthetic indices have been employed for this study: a Covering Index (1), that is the percentage of total area of a single cell $(A)$ occupied by the urbanized area $(a)$ resulting of the sum of all the patches in that cell; the Fractal Dimension (2) which equals 2 times the logarithm of the perimeter $p_{i}(\mathrm{~m})$ of a patch, divided by the logarithm of the area of the patch $a_{i}\left(\mathrm{~m}^{2}\right)$; the Degree of Landscape Division (3) resulting by the quadrate of the ratio between the area of a patch $a_{i}$, and the entire urbanized area $\mathrm{a}_{\text {tot }}$ in a cell.

$$
\begin{aligned}
& C I=\frac{\sum_{i=1}^{n} a_{i}}{A} \\
& F D=\frac{2 \ln *\left(0.25 * \sum_{i=1}^{n} p_{i}\right)}{\ln \sum_{i=1}^{n} a_{i}}
\end{aligned}
$$

$$
D L D=1-\sum_{i=1}^{n}\left(\frac{a_{i}}{a_{t o t}}\right)^{2}
$$

where $a_{i}=$ area of patch

$\mathrm{a}_{\text {tot }}=$ total urbanized area in a cell

$\mathrm{p}_{\mathrm{i}}=$ perimeter of patch

$A=$ area of a square cell of $200 \mathrm{~m}$

3.2.3 Analysis and texture classification: The calculation has been proportioned by using a grid with square mesh of $200 \mathrm{~m}$, 
which has been overlaid at the previously vectorialized classification. Then it was applied a clip between mesh and classification of impervious areas, and for each square cell it was computed the indices. It was also implemented a filter of spatial correlation of $200 \mathrm{~m}$, through the use of a buffer. The effectiveness of the filter consists in reaching a homogenization of values, based on the generalized concept that nearby objects tend to similarity. It also serves to avoid an unsuitable fragmentation of data. Once obtained the final results of indices for every impervious region in the cells, it was applied a cluster analysis to automatically classify homogeneous areas, consistently with those indicators and based on the conceptual models of texture types previously theorized: continuous, discontinuous, and scattered. Depending on these categories it was merged all the polygons belonging to the same class, and then exploded, to obtain the final database of polygons with their own morphological characteristics (Figure 7). Further analysis have been undertaken which aimed to quantify the amount of every typology of urban texture, and the proportions between them and the total amount of land consumption.

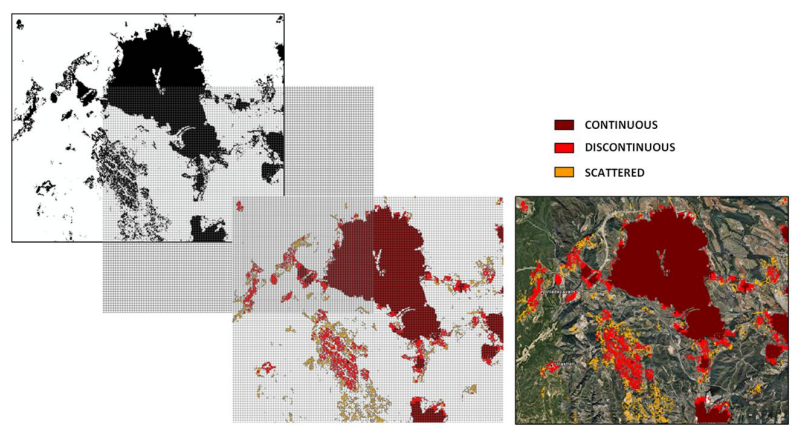

Figure 7. Result of cluster analysis about urban texture types

\section{CONCLUSIONS}

For collecting the necessary data to provide analysis of urban growth phenomena, the data which can be derived through remote sensing is inherently suited to offer essential information about the characteristics of different land cover categories at different spatial and temporary scales. The development of processing algorithms for satellite imagery and techniques for getting information, accurately and consistently, together with the development of analytical techniques and methods for obtaining indicators of specific attributes for urban growth modelling are essential tool to generate synthetic system to cover all the main aspects, morphological, environmental and socio-economical, about the dynamics of urban growth. The relevance of this work is the possibility to analyze those areas affected for high levels of land consumption, due to the urbanization, and which kind of urban texture is being more developed, it means, if the urban sprawl phenomena is leading the urban policies, or if the cities are following a typical Mediterranean model mostly based on the compactness.

\section{BIBLIOGRAPHY}

Crist E. P. and Cicone R. C., 1984, A physically-based transformation of Thematic Mapper data -- the TM Tasseled Cap, IEEE Trans. on Geosciences and Remote Sensing, GE-22: 256-263.

Crocetto N., Tarantino E., 2009. A class-oriented strategy for features extraction from multidate ASTER imagery. Remote Sensing, 1, 1171-1189.
Frenkel A., Ashkenazi M. 2008. Measuring urban srawl; how can we deal with it? Environment and Planning B: Planning and Design, 35 (1): 56-79

Huang, C., B. Wylie, L. Yang, C. Homer, and G. Zylstra. Derivation of a Tasseled Cap Transformation Based on Landsat 7 At-Satellite Reflectance. USGS EROS Data Center White Paper.

Huete, A.R., H. Liu, K. Batchily, and W. van Leeuwen, 1997. A Comparison of Vegetation Indices Over a Global Set of TM Images for EOS-MODIS. Remote Sensing of Environment 59(3):440-451.

Jaeger J. A. G. 2000. Landscape division, splitting index, and effective mesh size: new measures of landscape fragmentation. Landscape Ecology, 15: 115-130

Kasimu A., Tateishi R., 2010. Extracting area at risk of desertification using MODIS and geophysical data: in Xinjiang Uyghur Autonomous Region of China. International Conference of Multimedia Technology (ICMT).

Kaufman Y.J. and D. Tanre, 1996. Strategy for Direct and Indirect Methods for Correcting the Aerosol Effect on Remote Sensing: from AVHRR to EOS-MODIS. Remote Sensing of Environment, 55:65-79.

Lin W., Wang Q., Zha S., Li J., 2010. Construction and application of characteristic bands of typical land cover based on spectrum-photometric method. $18^{\text {th }}$ International Conference on Geoinformatics.

Richards J. A., 1999, Remote Sensing Digital Image Analysis, Springer-Verlag, Berlin, p. 240.

Qi J., Chehbouni A., Huete A. R., Kerr Y. H., Sorooshian S., 1994. A modified soil adjusted vegetation index. Remote Sensing of Environment, vol. 48, issue 2, pp.119-126.

XU H. Q., 2005. Fast information extraction of urban built-up land based on the analysis of spectral signature and normalized difference index. Geographical Research, vol. 24, pp. 311-318.

XU H. Q., 2006. Modification of normalized difference water index (NDWI) to enhance open water features in remotely sensed imagery. International Journal of Remote Sensing, vol. $27, \mathrm{n}^{\circ} 14$, pp. 3025-3033.

Zha Y., Ni S. X., Yang S., 2003. An effective approach to automatically extract urban land-use from TM imagery. Journal of Remote Sensing, vol.7, pp.37-40.

\section{ACKNOWLEDGEMENTS}

The authors of this paper acknowledge the research funding provided by the Spanish Ministry of Education and Science (SEJ2006-09630), the Spanish Ministry of Science and Innovation (CSO2009-09057), the Spanish Ministry of Development (E08/08), and the Spanish Ministry of Housing. Acknowledgements are also due to the European Union through the INTERREG IIIB Program (South Western Europe). For technical support the authors strongly acknowledge Montserrat Moix, Carlos Marmolejo, Jorge Cerda, staff members at Centre of Land Policy and Valuations (CPSV) of the Technical University of Catalonia (UPC) (Barcelona TECH). 